\title{
Effects of silencing key genes in the capsanthin biosynthetic pathway on fruit color of detached pepper fruits
}

\author{
Shi-Lin Tian ${ }^{1,2}$, Li Li ${ }^{2}$, Wei-Guo Chai ${ }^{3}$, Syed Noor Muhammad Shah ${ }^{1,4}$ and Zhen-Hui Gong ${ }^{1 *}$
}

\begin{abstract}
Background: There are many varieties of carotenoids in pepper fruits. Capsanthin is a red carotenoid that gives mature pepper fruits their red color. The red color in pepper fruits is regulated mainly by the genes capsanthin/ capsorubin synthase(Ccs), phytoene synthase(Psy), lycopene- $\beta$-cyclase(Lcyb) and $\beta$-carotene hydroxylase(Crtz). There has been very limited research work related to the development and change in the red color during fruit formation and when a certain gene or several genes are deleted. In this paper, we constructed viral vectors, using the tobacco rattle virus (TRV), to carry the target gene to infect detached pepper fruits, and observed the fruits' color change. We used real-time quantitative PCR to analyze the gene silencing efficiency. At the same time, HPLC was used to determine the content of capsanthin and carotenoids that are associated with capsanthin synthesis when key genes in the pepper fruits were silenced.

Results: These genes (CCS, Psy, Lcyb and Crtz) were individually silenced through virus induced gene silencing (VIGS) technology, and pepper fruits from red fruit cultivars showed an orange or yellow color. When several genes were silenced simultaneously, the fruit also did not show the normal red color. Gene expression analysis by real-time quantitative PCR showed 70-80\% efficiency of target gene silencing when using the VIGS method. HPLC analysis showed that the contents of carotenoids associated with capsanthin synthesis (e.g. $\beta$-carotene, $\beta$-cryptoxanthin or zeaxanthin) were decreased in varying degrees when silencing a gene or several genes together, however, the content of capsanthin reduced significantly. The synthesis of capsanthin was influenced either directly or indirectly when any key gene was silenced. The influence of the target genes on color changes in pepper fruits was confirmed via the targeted silencing of them.
\end{abstract}

Conclusions: VIGS was a good method to study the molecular mechanism of pepper fruit color formation. By using virus induced gene silencing technology, capsanthin synthesis genes in pepper fruits were silenced individually or simultaneously, and pepper fruit color changes were observed. This provides a platform to further explore the molecular mechanism of pepper fruit color formation.

Keywords: Tobacco rattle virus, VIGS, Detached fruit, Fruit color, Pepper (Capsicum annuum L.), Capsanthin

\section{Background}

Tobacco rattle virus (TRV) has straight tubular particles of two predominant lengths, the longer are about $190 \mathrm{~nm}$ and the shorter are 50 to $115 \mathrm{~nm}$, depending on the isolate. Normal particle-producing isolates (called M-type) have two species of genomic RNA, i.e. RNA1 and RNA2.

\footnotetext{
* Correspondence: zhgong@nwsuaf.edu.cn

'College of Horticulture, Northwest A\&F University, Yangling, Shaanxi 712100, P. R. China

Full list of author information is available at the end of the article
}

These are readily transmitted by inoculation with sap, and by nematodes in the family trichodoridae.

TRV is a useful vector because of its bipartite RNA. The RNA1 and RNA2 sequences of TRV can be used independently as vectors in plants and plant cells. A TRVRNA2 vector can be engineered to carry a heterologous nucleic acid for delivery into a plant. The TRV vector induces very mild symptoms, infects large areas of adjacent cells and silences gene expression in growing points. In addition, it is commonly used to enable gene identification. TRV is a positive-strand RNA virus with a bipartite

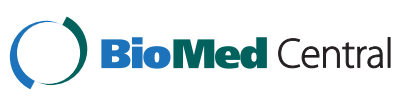


genome. Proteins encoded by RNA1 are sufficient for replication and movement within the host plant, while proteins encoded by RNA2 allow virion formation and nematode-mediated transmission between plants [1].

One of the most effective forms of plant defense against viruses is posttranscriptional gene silencing (PTGS). In this the plant's RNA-silencing machinery is activated and the virus will be subject to RNA silencing. Therefore, PTGS is an attractive endogenous process that can be exploited to study gene function. Virus-induced gene silencing (VIGS) is one of the most efficient approaches to activate the PTGS process. When a recombinant viral vector (VIGS vector) carrying a host-derived target gene sequence infects a plant, the TRV viral double-stranded RNAs are synthesized, leading to the activation of the antiviral RNA silencing pathway and the subsequent knockdown of the endogenous host gene. The VIGS technique is nowadays widely used to allow the transient interruption of gene function through a process similar to RNA interference [2]. The basis of the technique is a mechanism that is inherent in the plants for combating viruses [3,4]. Engineered viruses carrying one or more target genes are introduced into the plant. The double stranded RNA produced during virus replication triggers the degradation of any RNA with sequence similarity, including the endogenous transcripts of the target gene(s).

Pepper is an important vegetable crop, which enriches our diets. The pepper colors are mainly determined by chlorophyll, anthocyanin and carotenoid pigments; with carotenoids being responsible for colors in mature pepper fruits. Previous studies have shown that a range of genes are responsible for carotenoid formation and it is these that result in the varied colors of pepper fruits $[5,6]$. Capsanthin is a red carotenoid that gives mature pepper fruits their red color, and it is an end product in the pepper carotenoid biosynthesis pathway. The capsanthin biosynthetic pathway starts from geranylgeranyl diphospahate (GGPP), and then phytoene synthase (Psy) converts two molecules of GGPP to phytoene. After this, four desaturation reactions convert phytoene to lycopene, and the lycopene undergoes a cyclization reaction at both ends mediated by lycopene $\beta$-cyclase $(L c y b)$, thus producing $\beta$-carotene. $\beta$-carotene is then converted to $\beta$ cryptoxanthin and zeaxanthin with the reactions being triggered by $\beta$-carotene hydroxylase $(\mathrm{Crtz})$. Zeaxanthin is converted into antheraxanthin and violaxanthin when catalyzed by zeaxanthin expoxidase (Zep); then, antheraxanthin and violaxanthin are converted to capsanthin by Ccs and Zep [6]. The Psy, Lcyb, Crtz and Ccs genes that are involved in the capsanthin biosynthesis pathway have been cloned from pepper [7].These genes are directly involved in the red color of fruits $[8,9]$. The capsanthin and capsorubin pigments are responsible for the red color in pepper fruits, and they are regulated by $C c s$ gene. When the fruits starting to ripening, the Ccs gene begins to be expressed, which catalyzes zeaxanthin to be transformed into capsanthin [10]. The yellow color in pepper fruits is due to a Ccs gene deletion or Ccs mutation, which means that capsanthin cannot be synthesized [11]. The question raised here is whether pepper fruit colors are associated with Ccs gene expression or not. Therefore, it is important to understand the functions of the main genes that regulate the color development on peppers.

There has been very little research work on the color change in detached pepper fruits. We used virus-induced gene silencing (VIGS) technology to explore the molecular mechanism of color formation in the detached fruits. With the focus on the silencing of key genes involved in the capsanthin biosynthetic pathway and an exploration of the effects of different genes being removed on pepper fruit color formation.

\section{Results}

\section{Effects of certain genes being silenced on pepper fruit} color

The Ccs gene carried by the TRV viral vector was injected into detached fruits of Capsicum annuum cv. R15. Compared to the control fruits, different colors were observed in the fruits that had been treated with the TRV vector that had the Ccs gene, 15 days after inoculation (Figure 1). The color of the fruits injected with the TRV vector carrying the Ccs gene was from green to yellow (Figure 1d), while the control fruits were green to red color (Figure 1b). These results showed that a yellow fruit color is because of the silencing of the Ccs gene. We further confirmed these results with the TRV/00, in which the empty vector (TRV/00 = TRV1 and TRV2, no Ccs gene) was injected into detached pepper fruits, and the fruits were found to be the same color as the control fruits (Figure 1c). This confirmed that the yellow pepper fruit color is due to silencing of the Ccs gene (Figure 1).

We observed phenotypic changes in the pepper fruits using the VIGS with Psy, Lcyb and Crtz genes silenced. The color of the fruits was orange when the Psy gene was silenced (Figure 2), while yellow when the Lcyb and Crtz genes were silenced (Figures 3 and 4).

We used VIGS technology to determine the purpose of key genes involved in the color development in pepper fruits, and confirmed these changes by observing them in mature fruits.

\section{Changes in fruits' color during simultaneous silencing of several key genes}

We simultaneously silenced key genes to observe the effect of the genes deletion on the pepper fruits' color. Firstly, we simultaneously silenced two genes (Psy and $L c y b$ ), and obtained pepper fruits with a slightly orange color (Figure 5; Additional file 1: Table S1). Secondly, we 


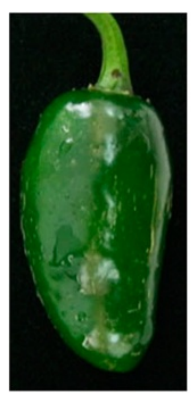

Before injection

(a)

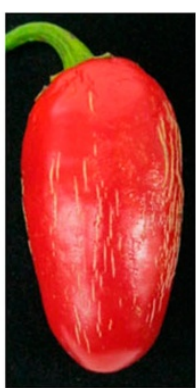

WT-Fruits

(b)

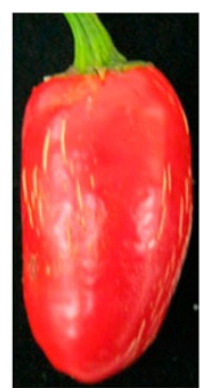

TRV/00

(c)

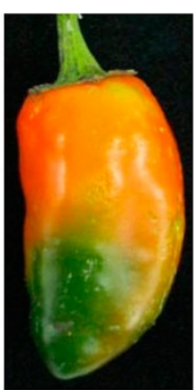

$\mathrm{TRV} / \mathrm{Ccs}$

(d)

Figure 1 Phenotype changes in pepper fruits with Ccs gene silencing via VIGS. (a) The fruit on the 35th DAA when it is still in the green mature stage; (b) WT-fruit: the phenotype of fruits that were not injected with the TRV vector carrying the Ccs gene after fruits were kept in growth chambers for 15d; (c) TRV/00: the phenotype of fruits that were injected with the TRV empty vector after fruits were kept in growth chambers for 15d; and (d) TRV/Ccs: the phenotype of fruits that were injected with the TRV vector carrying the Ccs gene after fruits were kept in growth chambers for $15 \mathrm{~d}$.

simultaneously silenced three genes (Psy, Lcyb and $C r t z)$, and observed that the fruits' color went from green to yellow (Figure 6). Thirdly, we simultaneously silenced four key genes (Ccs, Psy, Lcyb and Crtz), and observed that the pepper fruits' color went from green to bright yellow (Figure 7; Additional file 1: Table S1). These results showed that single gene silencing and multi-gene silencing had different effects on the pepper fruit color, which revealed that several genes had synergistic effects on fruit color formation.

\section{Changes in target genes' expression in pepper fruits through VIGS technology}

To better understand the relationship between the fruits' color and gene expression, we extracted RNA from normal fruits, TRV empty vector injected fruits and gene silenced fruits. Total RNA was purified and first-strand cDNA was synthesized by reverse transcriptase. Then real-time quantitative PCR was used to determine the genes' expression.

Firstly, we looked at the phenotypic fruit color variation when a single target gene was silenced. From Figure 8 it could be seen that when the Ccs gene was silenced, there were no significant differences in the Ccs gene expression level of the WT and TRV/00 groups, the Ccs gene expression level in the TRV/Ccs group was significantly decreased compared with the WT group and the Ccs gene expression level of the TRV/Ccs group was about $20 \%$ that in the WT and TRV/00 groups. All the other genes (either WT and TRV/00 groups or TRV/Ccs) showed normal expressions. This demonstrated that the yellow phenotype of the pepper fruits was due to the Ccs gene being silenced when the TRV carrying the Ccs gene infected the detached pepper fruits (Figure 8). Similarly, when the Psy gene was silenced, there were no significant differences in the Psy gene expression level of the WT and TRV/00 groups, the

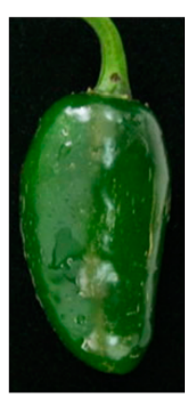

Before injection

(a)

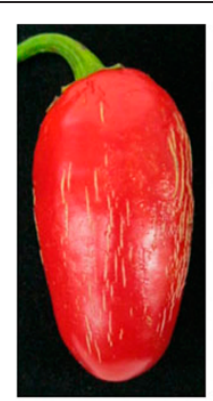

WT-Fruits

(b)

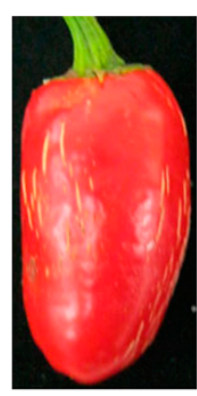

TRV/00

(c)

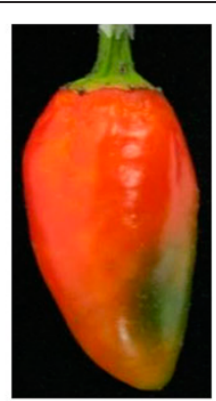

TRV/Psy

(d)

Figure 2 Phenotype changes in pepper fruits with Psy gene silencing via VIGS. (a) The fruit on the 35th DAA when it is still in the green mature stage; (b) WT-fruit: the phenotype of fruits that were not injected with the TRV vector carrying the Psy gene after fruits were kept in growth chambers for 15d; (c) TRV/00: the phenotype of fruits that were injected with the TRV empty vector after fruits were kept in growth chambers for 15d; and (d) TRV/Psy: the phenotype of fruits that were injected with the TRV vector carrying the Psy gene after fruits were kept in growth chambers for $15 \mathrm{~d}$. 


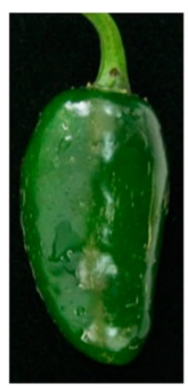

Before injection

(a)

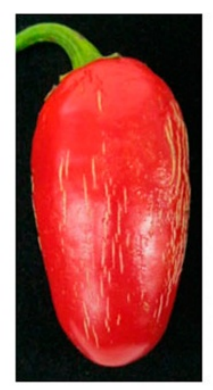

WT-Fruits

(b)

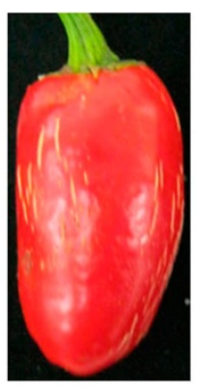

TRV/00

(c)

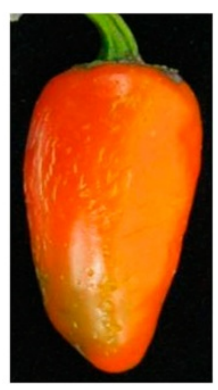

TRV/Lcyb

(d)

Figure 3 Phenotype changes in pepper fruits with Lcyb gene silencing via VIGS. (a) The fruit on the 35th DAA when it is still in the green mature stage; (b) WT-fruit: the phenotype of fruits that were not injected with the TRV vector carrying the Lcyb gene after fruits were kept in growth chambers for 15d; (c) TRV/00: the phenotype of fruits that were injected with the TRV empty vector after fruits were kept in growth chambers for 15d; and (d) TRV/Lcyb: the phenotype of fruits that were injected with the TRV vector carrying the Lcyb gene after fruits were kept in growth chambers for $15 d$.

Psy gene expression level in the TRV/Psy group was significantly decreased compared with the WT group and the Psy gene expression level of the TRV/Psy group was about $20 \%$ that in the WT and TRV/00 groups. All other genes (either WT and TRV/00 groups or TRV/Psy groups) showed normal expressions. This demonstrated that the orange phenotype of the pepper fruits was due to the Psy gene being silenced when the TRV carrying the Psy gene infected the detached pepper fruits (Figure 9). For the TRV/Lcyb and TRV/Crtz, the expression of the Lcyb, Crtz and other genes in the WT group, TRV/00 group and TRV/target genes group were similar. That is, the deep yellow phenotype of the pepper fruits was due to the $L c y b$ gene or Crtz gene being silenced when the TRV carrying the Psy gene or Crtz gene infected the detached pepper fruits (Figures 10 and 11).
Secondly, we looked at the phenotype of the fruits' color variation when several key genes were simultaneously silenced. When the Psy and Lcyb genes were silenced simultaneously, there were no significant differences in the Psy and $L c y b$ genes expression levels of the WT and TRV/00 groups, the Psy and Lcyb genes expression levels in the TRV/Psy/Lcyb group were significantly decreased compared with the WT group and the Psy and Lcyb genes expression levels of the TRV/Psy/Lcyb group were about $20 \%-30 \%$ that of the WT and TRV/00 groups. All other genes, for example, $C c s$ and $C r t z$ genes, (either the WT and TRV/00 groups or TRV/PSY/Lcyb group) showed normal expressions.This demonstrated that the slightly orange phenotype of the pepper fruits was due to the Psy and Lcyb genes being silenced when the TRV carrying the Psy and $L c y b$ genes infected the detached pepper fruits (Figure 12).

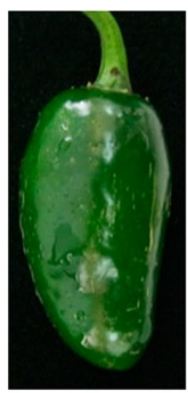

Before injection

(a)

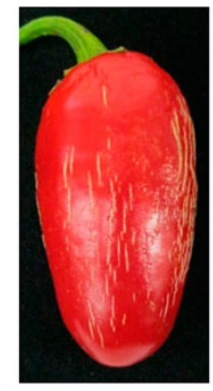

WT-Fruits

(b)

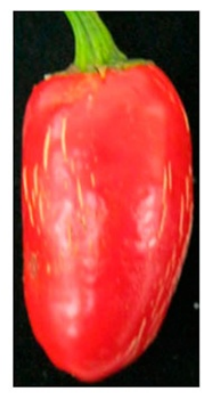

TRV/00

(c)

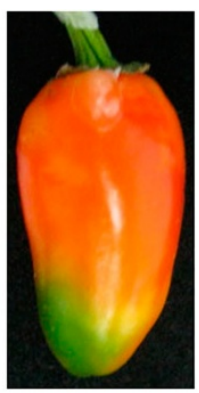

$\mathrm{TRV} / \mathrm{Crtz}$

(d)

Figure 4 Phenotype changes in pepper fruits with Crtz gene silencing via VIGS. (a) The fruit on the 35th DAA when it is still in the green mature stage; (b) WT-fruit: the phenotype of fruits that were not injected with the TRV vector carrying the Crtz gene after fruits were kept in growth chambers for 15d; (c) TRV/00: the phenotype of fruits that were injected with the TRV empty vector fruit after fruits were kept in growth chambers for 15d; and (d) TRV/Crtz: the phenotype of fruits that were injected with the TRV vector carrying the Crtz gene after fruits were kept in growth chambers for $15 \mathrm{~d}$. 


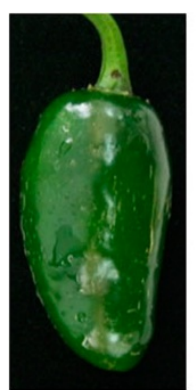

Before injection

(a)

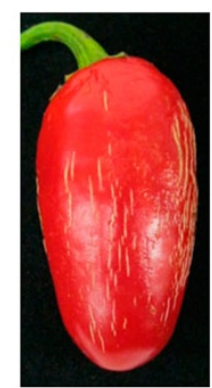

WT-Fruits

(b)

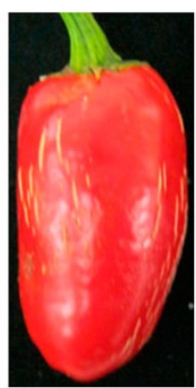

TRV $/ 00$

(c)

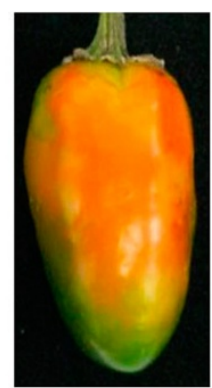

TRV/Psy/Lcyb

(d)

Figure 5 Phenotype changes in pepper fruits with simultaneous Psy and Lcyb genes silencing via VIGS. (a) The fruit on the 35th DAA when it is still in the green mature stage; (b) WT-fruit: the phenotype of fruits that were not injected with the TRV vector carrying the target gene after fruits were kept in growth chambers for 15d; (c) TRV/00: the phenotype of fruits that were injected with the TRV empty vector after fruits were kept in growth chambers for 15d; and (d) TRV/Psy/Lcyb: the phenotype of fruits that were injected with the TRV vector carrying the Psy and Lcyb genes after fruits were kept in growth chambers for $15 \mathrm{~d}$.

When the Psy, Lcyb and Crtz genes were silenced simultaneously, there were no significant differences in the Psy, Lcyb and Crtz genes expression levels of the WT and TRV/00 groups, the Psy, Lcyb and Crtz genes expression levels in the TRV/Psy/Lcyb/Crtz group were significantly decreased compared with the WT group but the Psy, Lcyb and Crtz genes expression levels of the TRV/Psy/Lcyb/Crtz group were about 20\%-30\% that in the WT and TRV/00 groups. All other genes, for example, the Ccs gene, (either the WT and TRV/00 groups or TRV/Psy/Lcyb/Crtz) showed normal expressions. This demonstrated that the yellow phenotype of the pepper fruits was due to the Psy, Lcyb and Crtz genes being silenced when the TRV carrying the Psy, $L c y b$ and $C r t z$ genes infected the detached pepper fruits (Figure 13).
When the Psy, Lcyb, Crtz and Ccs genes were silenced simultaneously, there were no significant differences in the Psy, Lcyb, Crtz and Ccs genes expression levels in the WT and TRV/00 groups, the Psy, Lcyb, Crtz and Ccs genes expression levels in the TRV/Psy/Lcyb/Crtz/Ccs group were significantly decreased compared with the WT group and the Psy, Lcyb, Crtz and Ccs genes expression levels in the TRV/Psy/Lcyb/Crtz/Ccs group were about 20\%-30\% that of the WT and TRV/00 groups. This demonstrated that the bright yellow phenotype of the pepper fruits was due to the Psy, Lcyb, Crtz and Ccs genes being silenced when the TRV carrying the Psy, Lcyb, Crtz and Ccs genes infected the detached pepper fruits (Figure 14).

When considering the above results it could be seen that when the TRV vector carrying the target gene was injected into detached fruits the expression levels of the

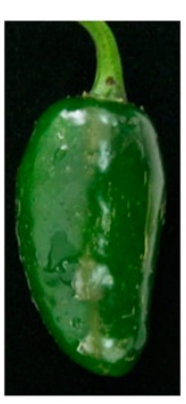

Before injection

(a)

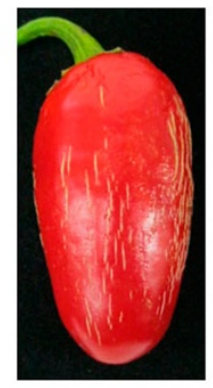

WT-Fruits

(b)

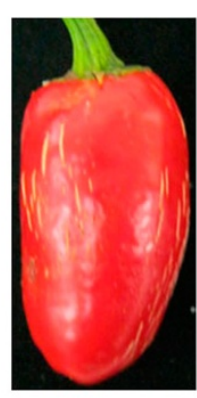

TRV $/ 00$

(c)

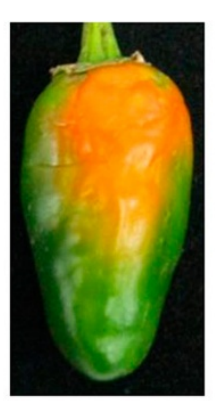

TRV/Psy/Lcyb/Crtz

(d)

Figure 6 Phenotype changes in pepper fruits with simultaneous Psy, Lcyb and Crtz genes silencing via VIGS. (a) The fruit on the 35th DAA when it is still in the green mature stage; (b) WT-fruit: the phenotype of fruits that were not injected with the TRV vector carrying the target gene after fruits were kept in growth chambers for 15d; (c) TRV/00: the phenotype of fruits that were injected with the TRV empty vector after fruits were kept in growth chambers for 15d; and (d) TRV/Psy/Lcyb/Crtz: the phenotype of fruits that were injected with the TRV vector carrying the Psy, Lcyb and Crtz genes after fruits were kept in growth chambers for 15d. 


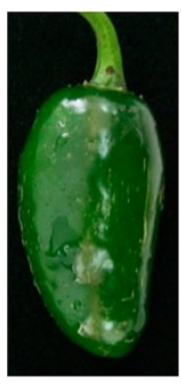

Before injection

(a)

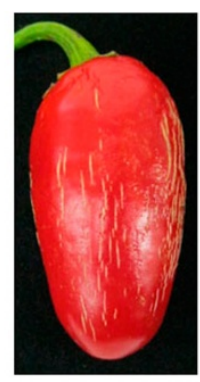

WT-Fruits

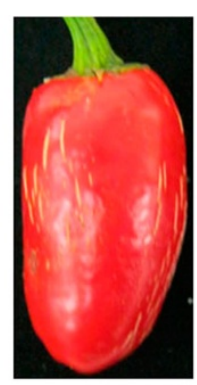

TRV/00

(c)

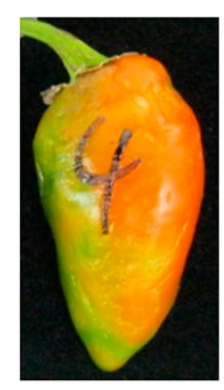

$\mathrm{TRV} / \mathrm{Psy} / \mathrm{Lcyb} / \mathrm{Crtz} / \mathrm{Ccs}$

(d)

Figure 7 Phenotype changes in pepper fruits with simultaneous Psy, Lcyb, Crtz and Ccs genes silencing via VIGS. (a) The fruit on the 35th DAA when it is still in the green mature stage; (b) WT-fruit: the phenotype of fruits that were not injected with the TRV vector carrying the target gene after fruits were kept in growth chambers for 15d; (c) TRV/00: the phenotype of fruits that were injected with the TRV empty vector after fruits were kept in growth chambers for 15d; and (d) TRV/Psy/Lcyb/Crtz/Ccs: the phenotype of fruits that were injected with the TRV vector carrying Psy, Lcyb, Crtz and Ccs genes after fruits were kept in growth chambers for $15 \mathrm{~d}$.

genes were $20 \%-30 \%$ those of the levels in the normal fruits and those injected with an empty vector (Figures 8 , $9,10,11,12,13$ and 14). This showed that a $70 \%-80 \%$ efficiency of target gene silencing was achieved. These results proved that gene silencing caused fruit color changes. Therefore, the target gene silencing was a cause of fruit color change.

\section{Changes in composition of carotenoids in pepper fruits when a gene or some genes were silenced}

The phenomenon of high or low target gene expression levels were not enough for an interpretation of the phenotype of the pepper fruits' color variation. Therefore, we determined the carotenoids' composition in pepper fruits using the HPLC method (Figure 15). An HPLC system that

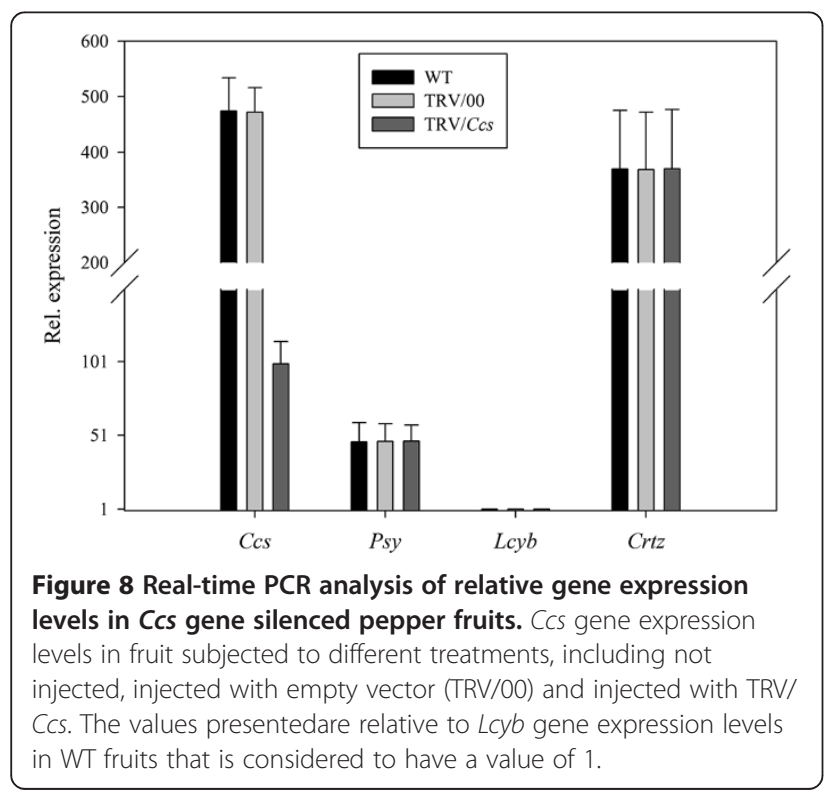

was able to resolve the $\beta$-carotene, $\beta$-cryptoxanthin, zeaxanthin and capsanthin, and determine their levels via detection at an absorbance of $454 \mathrm{~nm}$ was utilized. Additional file 2: Figure S1, Additional file 3: Figure S2, Additional file 4: Figure S3, Additional file 5: Figure S4, Additional file 6: Figure S5, Additional file 7: Figure S6, Additional file 8: Figure S7, Additional file 9: Figure S8, Additional file 10: Figure S9 contain the HPLC profiles.

We knew that $\beta$-carotene, $\beta$-cryptoxanthin, zeaxanthin and capsanthin are the primary ingredients in the pepper fruits' carotenoids biosynthetic pathway. These carotenoid ingredients would be affected when a gene or several genes were silenced. From Figure 15, we could see that the WT and TRV/00 had no obvious differences in the carotenoids' compositions. And we also could identify that

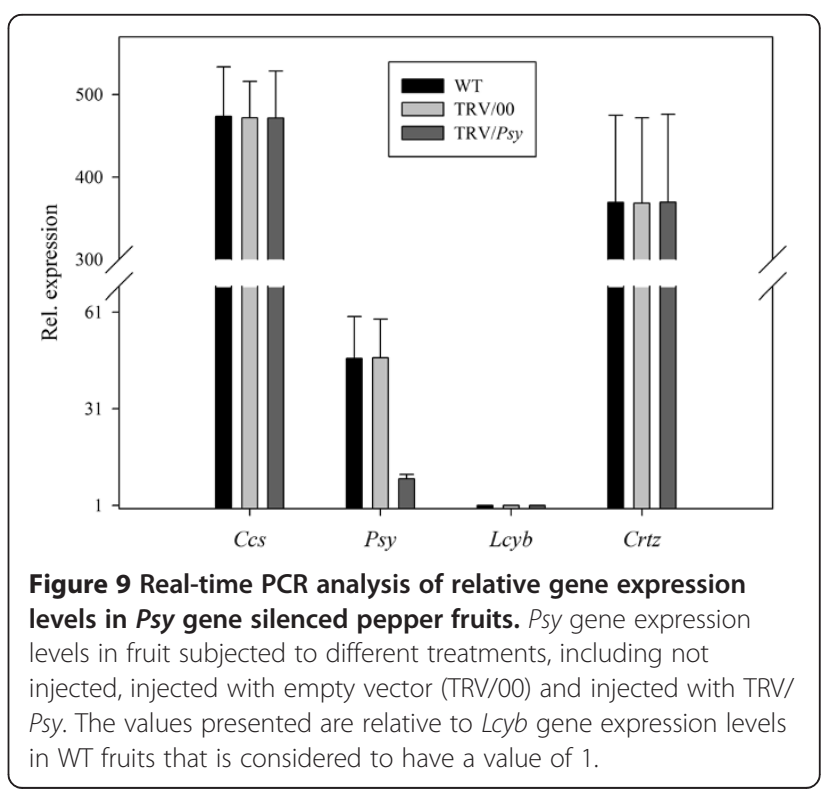




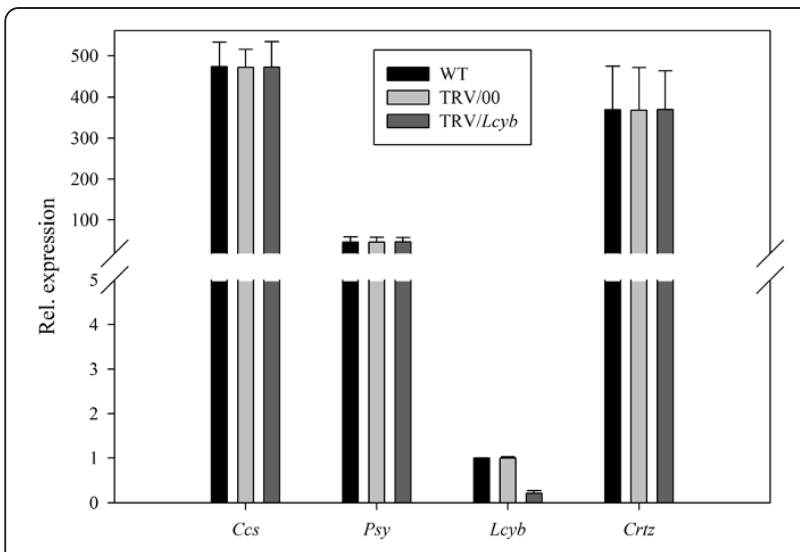

Figure 10 Real-time PCR analysis of relative gene expression levels in $L c y b$ gene silenced pepper fruits. $L c y b$ gene expression levels in fruit subjected to different treatments, including not injected, injected with empty vector (TRV/00) and injected with TRV/Lcyb. The values presented are relative to $L c y b$ gene expression levels in $W T$ fruits that is considered to have a value of 1 .

the levels of the metabolic intermediates (e.g. $\beta$-carotene, $\beta$-cryptoxanthin or zeaxanthin) in the capsanthin biosynthetic pathway had different rate reductions when one or several key genes were silenced in pepper fruit, but there was a slight noticeable change in the content of capsanthin. Therefore, to obtain a greater understanding the results need to be considered in greater detail. First of all, when silencing the Ccs gene, the levels of $\beta$-carotene, $\beta$ cryptoxanthin, zeaxanthin and capsanthin reduced than those of the WT. however, the capsanthin content of the fruits in which $C c s$ was silenced reduced significantly. This demonstrated that the fruit color change was caused by silencing the Ccs gene. Secondly, when silencing the Psy gene, compared with the WT, the content of $\beta$-carotene,

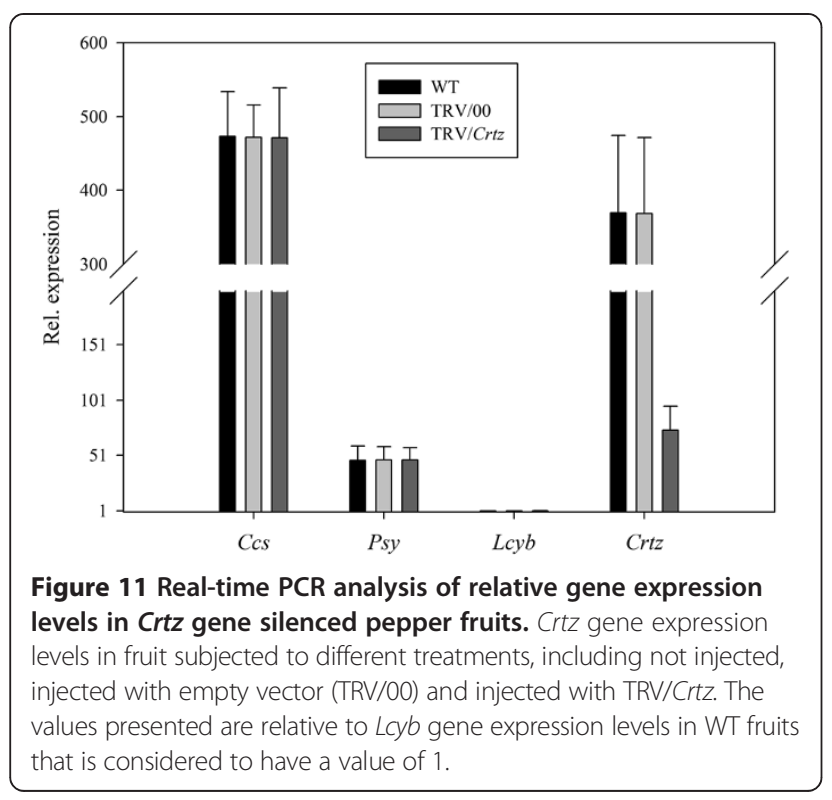

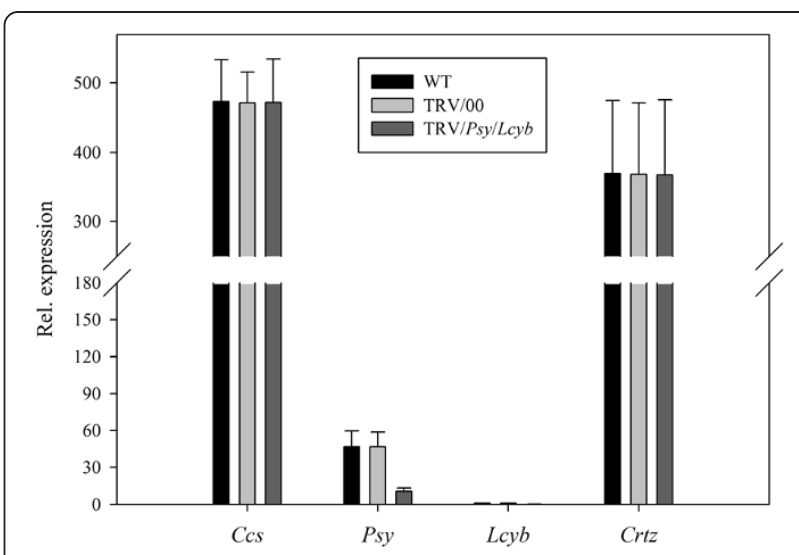

Figure 12 Relative gene expression levels with simultaneously silenced Psy and Lcyb genes in pepper fruits via VIGS. Psy gene expression levels in fruits subjected to different treatments, including not injected, injected with empty vector (TRV/00) and injected with TRV/Psy/Lcyb. Lcyb gene expression levels in fruits subjected to different treatments, including not injected, injected with empty vector (TRV/00) and injected with TRV/Psy/Lcyb. The values presented are relative to Lcyb gene expression levels in WT fruits that is considered to have a value of 1 .

$\beta$-cryptoxanthin, zeaxanthin and capsanthin in the TRV/ Psy fruits reduced significantly. This indicated that the intermediate products of the capsanthin biosynthetic pathway were negatively affected by silencing the Psy gene, which resulted in the content of capsanthin being reduced. This resulted in a significant difference in the fruit color of normal and silenced fruits. Thirdly, when

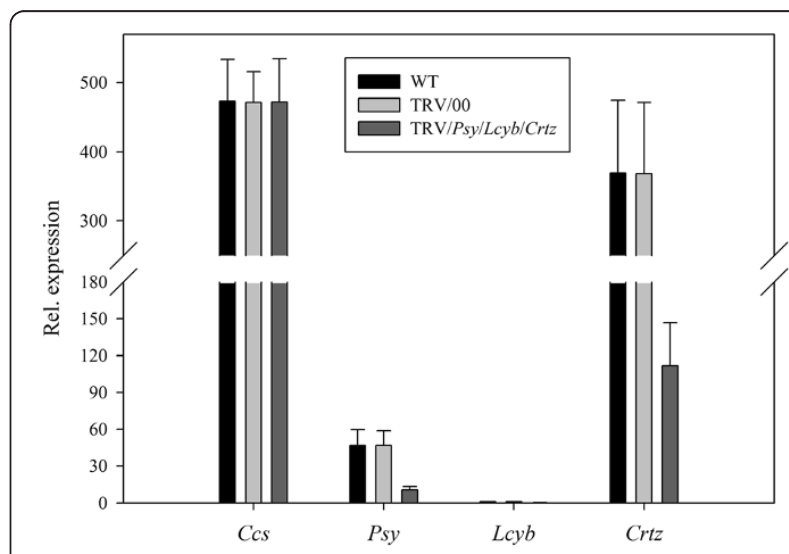

Figure 13 Relative gene expression levels with simultaneously silenced Psy,Lcyb and Crtz genes in pepper fruits via VIGS. PSY gene expression levels in fruits subjected to different treatments, including not injected, injected with empty vector (TRV/00) and injected with TRV/Psy/Lcyb/Crtz. Lcyb gene expression levels in fruits subjected to different treatments, including not injected, injected with empty vector (TRV/00) and injected with TRV/Psy/Lcyb/Crtz. Crtz gene expression levels in fruits subjected to different treatments, including not injected, injected with empty vector (TRV/00) and injected with TRV/Psy/Lcyb/Crtz. The values presented are relative to Lcyb gene expression levels in WT fruits that is considered to have a value of 1. 


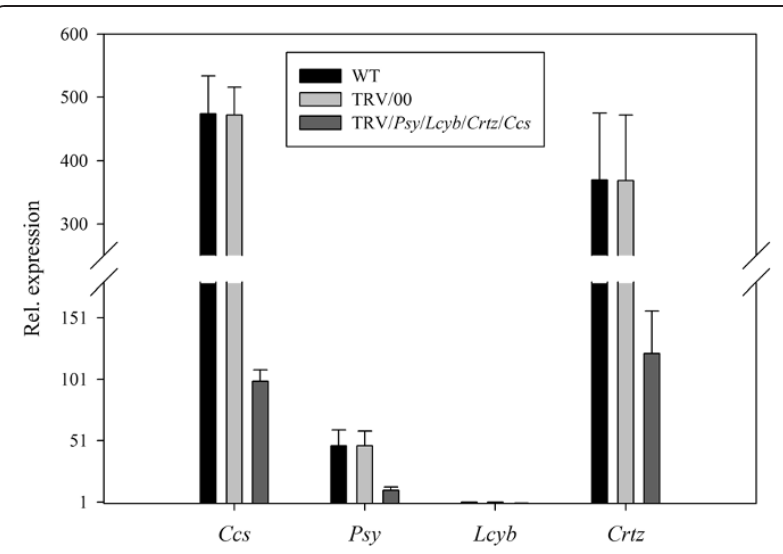

Figure 14 Relative gene expression levels with simultaneously silenced Psy, Lcyb, Crtz and Ccs genes in pepper fruits via VIGS. Psy gene expression levels in fruits subjected to different treatments, including not injected, injected with empty vector (TRV/00) and injected with TRV/Psy/Lcyb/Crtz/Ccs. Lcyb gene expression levels in fruits subjected to different treatments, including not injected, injected with empty vector (TRV/00) and injected with TRV/Psy/Lcyb/Crtz/Ccs. Crtz gene expression levels in fruits subjected to different treatments, including not injected, injected with empty vector (TRV/00) and injected with TRV/Psy/Lcyb/Crtz/Ccs. Ccs gene expression levels in fruits subjected to different treatments, including not injected, injected with empty vector (TRV/00) and injected with TRV/Psy/Lcyb/Crtz/Ccs. The values presented are relative to $L c y b$ gene expression levels in WT fruits that are considered to have a value of 1 .

silencing the $L c y b$ gene, compared with the WT, the content of $\beta$-carotene, $\beta$-cryptoxanthin and capsanthin in the TRV/Lcyb fruits reduced at different rates, and the content of capsanthin decreased significantly by comparing with the control. Fourthly, when the Crtz gene was silenced, compared with the WT, the content of $\beta$-carotene, $\beta$-cryptoxanthin and zeaxanthin decreased slightly, while the content of capsanthin decreased significantly. Finally,

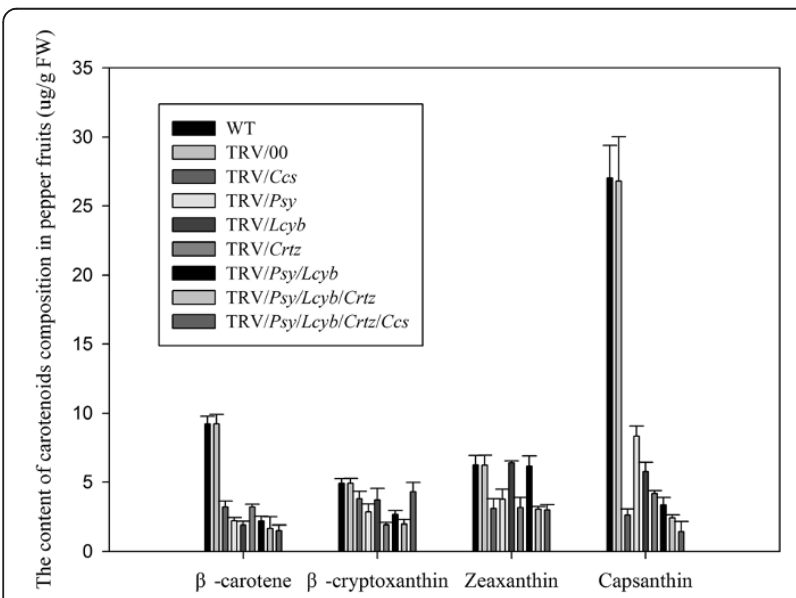

Figure 15 Carotenoids content in pepper fruits. WT: fruits not injected and TRV/00: fruits injected with empty vector. Values + SD of three independent biological replicates. when silencing several key genes, we can see that the content of $\beta$-carotene, $\beta$-cryptoxanthin and zeaxanthin decreased in different degrees, and the content of capsanthin decreased significantly among these metabolites. These results indicated that there was a significant reduction in the capsanthin content whether a single gene or several genes were simultaneously silenced. This showed that the change in fruit color was due to abnormal expression of these key genes, and this caused the content of the intermediate metabolite (e.g. $\beta$-carotene, $\beta$-cryptoxanthin or zeaxanthin) in the capsanthin biosynthetic pathway to be reduced. Eventually, this affected the normal synthesis of capsanthin, which resulted in the fruit color not becoming the normal red (Figure 15).

\section{Discussion}

VIGS is a quick method for target gene silencing to produce phenotype changes; therefore, VIGS can be used to quickly identify a gene's function [12]. TRV as a VIGS vector is able to penetrate the parts of a plant that are growing. This means that the infections could be spread by cell division as well as transport. As a result, infections will be more uniform and there would be less of a dilution effect from non-infected cells on the silencing. In addition, TRV-induced silencing could be initiated in undifferentiated growing point cells and the silencing would be masked by gene expression in non-infected cells due to the target gene expression prior to infection.

However, the silencing effect remains transient in the majority of cases and the timing of its appearance as well as its duration is species-specific. For example, the barley stripe mosaic virus (BSMV)-induced a VIGS effect in barley that lasted 1-2 weeks and TRV mediated silencing in california poppy (Eschscholzia californica) was lost after 16 weeks, while apple latent spherical virus (ALSV)-mediated silencing was maintained in soybean throughout the plant's life and was even transmitted to the next generation [13-15]. Another challenge when using this technique is a variation in the level of penetration in vegetative and reproductive tissue that requires a larger number of plants to be screened for phenotypes. Silencing effects are often found in dividing sectors of the plant or restricted to plant organs formed from a few consecutive nodes [13]. This study demonstrated that TRV-induced VIGS effects in detached pepper fruits lasted for 30 days, which was enough to determine the relationship between the genes and fruit color.

It could be observed from Figure 15 that in the WT group; when the fruits were ripe the major pigment was capsanthin that was present in much greater amounts than the other carotenoids. The higher the capsanthin content in the pepper fruits, the deeper the red color. Next we analyzed the causes of fruit color formation when key genes were silenced by VIGS. 
Firstly, we analyzed the causes of the fruits' color formation when a single target gene was silenced. By analyzing gene expression levels and the composition of the carotenoids, it was found that when the Ccs gene had a low expression level and the capsanthin synthesis decreased, the pepper fruit color was yellow when the Ccs gene was silenced. After Psy gene silencing, the Psy gene also had a low expression level and capsanthin synthesis decreased, but the level of capsanthin was higher in the TRV/Psy group than the TRV/Ccs group, so the pepper fruit color was orange when the Psy gene was silenced. Similarly, the TRV/Lcyb group and TRV/Crtz group caused a yellow fruit color after the Psy gene or Crtz gene was silenced.

Secondly, we analyzed the causes of the fruits' color formation when several key genes were silenced simultaneously. By analyzing gene expression levels and the composition of the carotenoids, it was found that the Psy and Lcyb genes had low expression levels and capsanthin synthesis decreased (the capsanthin content of the TRV/Psy/Lcyb group was far lower than that of the WT group) when the Psy and Lcyb genes were silenced simultaneously, which meant that the pepper fruit color was yellow. After the Psy, Lcyb and Crtz genes were silenced simultaneously, the Psy, Lcyb and Crtz genes also had low expression levels and the capsanthin synthesis decreased (the capsanthin content of the TRV/Psy/Lcyb/ $\mathrm{Crtz}$ group was far lower than that of the WT group), so the pepper fruit was yellow. Similarly, when the Psy, $L c y b, C r t z$ and $C c s$ genes were silenced simultaneously, the Psy, Lcyb, Crtz and Ccs genes also had low expression levels and the capsanthin synthesis decreased (the capsanthin content of the TRV/Psy/Lcyb/Crtz/Ccs group was far lower than that of the WT group), so the phenotype of the fruit was yellow (Figure 15).

The focus of previous studies has been the opposing genetic characters of red and yellow, and they determined that red is dominant and controlled by a single gene at the $y$ locus of the sixth chromosome $[11,16]$. The mature fruit color of the pepper is determined by capsanthin. Researchers have found that capsanthin is synthesized by the enzyme capsanthin-capsorubin synthase $(C c s)$. Ha et al [5] determined that the Ccs gene was not present in yellow pepper. Sequence analyses of the $C c s$ gene revealed two structural mutations in yellow peppers that are a result of either a premature stop-codon or a frame-shift. A Ccs transcript was not detectable in yellow peppers. The deletion of the Ccs gene is not responsible for the yellow ripening in Capsicum [5].

Previous studies related to pepper fruit color had a focus on $C c s$ gene deletion or mutation, while other key genes related to fruit color change were rarely studied. This study's experimental results revealed that the fruit color becoming yellow and orange was not only confined to Ccs gene silencing but it was also related to Psy, Lcyb and Ctrz genes silencing. This demonstrates that there is still a lot of work to be done on the fruit color genes. VIGS technology can be used to analyze gene function, and our experiments analyzed genes related to fruit color in detached fruit. As genes were silenced, the pepper fruits changed their color resulting in many different colors. Still, it needs to explore whether this is the result of the action of one gene or several genes, which will be solved in the future. VIGS technology was used with detached fruits, which provided reliable information about the relationship between genes and fruit color formation and gave quick results, which was important.

\section{Conclusions}

By using VIGS technology, we determined that there was a relationship between changes in pepper fruit color and the target genes' (Psy, Ccs, Lcyb and Crtz) silencing; while, single gene and multi-gene silencing had different effects on fruit color, as determined from the data presented in this paper. Compared with capsanthin, the levels of $\beta$ carotene, $\beta$-cryptoxanthin or zeaxanthin were very low in pepper fruits. In addition, the content of capsanthin decreased significantly after a single gene or a group of genes were silenced. Silencing any key gene would either directly or indirectly influence synthesis of capsanthin. This led to the pepper fruits' color changes. VIGS technology was used in detached fruits, which simplified the research process of studying the genes related to pepper fruit color changes. This gave a better platform to understand the relationship between colorful pepper fruits and the genetic regulation.

\section{Methods}

\section{Experimental material}

Seeds of Capsicum annuum cv. R15 (a tolerant storage cultivar) were provided by the Capsicum Research Group, College of Horticulture, Northwest A\&F University, P.R. China.

\section{Pre-sowing treatment of pepper seeds}

To break the dormancy of the pepper seeds, they were treated with hot water $\left(55^{\circ} \mathrm{C}\right)$ for $20 \mathrm{~min}$, and then soaked in water for $5 \mathrm{~h}$ at $28^{\circ} \mathrm{C}$. The seeds were then covered with a wet cotton cloth and placed in the dark in a growth chamber. Seeds started to germinate after four days and were transferred to pots to be raised as seedlings.

\section{Plant growth conditions and sample collection}

Once the seedlings had 8-10 true leaves they were taken and transplanted into plastic high-tunnels under natural field conditions. The fruits (all same age) on the 35th 
day after anthesis (green mature stage) were picked and transferred to the laboratory for the VIGS experiment.

\section{Virus vector construction}

Tobacco rattle virus (TRV) has bipartite RNA. Its RNA1 and RNA2 sequences can be used independently as vectors in plants and plant cells. The TRV-RNA2 vector can carry heterologous nucleic acid for delivery into a plant. A schematic representation of the TRV vector is shown in Additional file 11: Figure S10. According to the structure of the TRV, primers were designed that carried the $\mathrm{BamHI}$ site upstream and the Kpn I site downstream and they transferred the target genes into the TRV vector (Additional file 12: Table S2).

VIGS was performed in pepper cultivar R15 using the TRV-based VIGS technique. Four fragments from the 3' ends of the Ccs, Psy, Lcyb and Crtz open reading frames were cloned into the pTRV2 vector, and used to generate the pTRV2/Ccs, pTRV2/Psy, pTRV2/Lcyb and pTRV2/ $\mathrm{Crtz}$ vectors (Additional file 13: Figure S11), while the empty vector (pTRV/00) was used as a negative control.

\section{Cloning of target gene fragments}

The vector gene fragment size requirement was 150$500 \mathrm{bp}$ for gene silencing expression; primers for the PCR were designed according to GenBank (http://www.ncbi. nlm.nih.gov/genbank) using carotenoid-related genes (Additional file 12: Table S2). The total RNA of the fruits was purified with Trizol and submitted to cDNA synthesis, after the cDNA was used as a template for PCR amplification, agarose gel electrophoresis of PCR products was conducted and target gene fragments were recovered using a DNA extraction kit; and, the recovered products were ligated into a cloning vector pMD19-T using T4 DNA ligase at $16^{\circ} \mathrm{C}$ overnight and then transformed into Escherichia coli DH5a. Then if the PCR had been successful as determined by the recovery of recombinant plasmid (Additional file 14: Figure S12), the gene silencing vectors could be produced from the gene fragments. The plasmids pTRV2 and PMD19-T carrying the target genes were digested individually with BamHI and KpnI, and then the pTRV2 restriction fragments and target gene fragments were ligated together (Additional file 15: Figure S13).

\section{Genetic transformation of agrobacterium}

The pTRV1 and pTRV2 vectors were introduced into the Agrobacterium strain GV3101 by the freeze-thaw method [17]. The detection of bacteria in culture was confirmed by PCR, and the bacteria culture was stored for use in further experiments.

\section{Virus-induced gene silencing (VIGS)}

The pTRV1, pTRV2, pTRV2/Ccs, pTRV2/Psy, pTRV2/ $L c y b$ and pTRV2/Crtz vectors were mixed with the Agrobacterium tumefaciens strain GV3101 in a 1:1 ratio. The culture of Agrobacterium inocula containing pTR1 and pTRV2/00, pTRV2/Ccs, pTRV2/Psy, pTRV2/Lcyb and pTRV2/Crtz $\left(\mathrm{OD}_{600}=1.0\right)$ were injected into detached pepper fruits using a $1.0 \mathrm{ml}$ sterilized syringe without a needle. The composition of the TRV vector that had several genes silence simultaneously wasTRV/Ccs, TRV/Psy, $\mathrm{TRV} / L c y b$ and TRV/Crtz in a 1:1 ratio. Specifically, the TRV/Psy/Lcyb/Crtz/Ccs was made up of TRV/Ccs, TRV/ Psy, TRV/Lcyb and TRV/Crtz in a 1:1 ratio; the TRV/ $P s y / L c y b / C r t z$ was made up of TRV/Psy, TRV/Lcyband TRV/Crtzin a 1:1 ratio; and the TRV/Psy/Lcyb was made up of TRV/Psy and TRV/Lcyb in a 1:1 ratio. The treated fruits (TRV/00, TRV/Ccs, TRV/Psy, TRV/Lcyb and TRV/ $\mathrm{Crtz}$ ) were used for the respective gene analyses 15 days after inoculation.

\section{TRV virus vector inoculation in fruits}

Before treatments, the fruits were carefully washed with tap water and then a further three times with distilled water and dried at room temperature. Then before being placed in a sterilized laminar flow hood, the fruits had their stalks sealed with melted wax. The fruits were then sterilized in $75 \%$ alcohol for 30 seconds, and washed with sterilized distilled water three times. A small hole was made at the base of the fruits' stalks and $0.5 \mathrm{ml}$ of the TRV virus vector culture was injected into the fruits with a $1 \mathrm{ml}$ sterilized syringe without needle.

The fruits were placed on sterilized filter papers on a stainless steel plate and covered with food grade cling-film wrap. The plate was placed in a dark chamber $\left(18^{\circ} \mathrm{C}\right.$ and $35 \% \mathrm{RH})$ for two days. After two days the treated fruits were transferred into a growth chamber at $23^{\circ} \mathrm{C} / 20^{\circ} \mathrm{C}$ with a $16 \mathrm{~h}$ light $/ 8 \mathrm{~h}$ dark photoperiod cycle at $35 \%$ relative humidity. The control fruits (TRV/00) and silenced fruits (TRV/Ccs, TRV/Psy, TRV/Lcyb and TRV/Crtz) were respectively used for gene analysis 15 days after inoculation.

\section{RNA isolation and qRT-PCR analysis}

Total RNA was extracted from the normal fruits (control) and gene silenced fruits using the Trizol (Invitrogen) method [18]. The concentration of total RNA was measured by a spectrophotometer using a NanoDrop instrument (Thermo Scientific NanoDrop 2000C Technologies, Wilmington, USA), and the purity was assessed using the A260/280 and A260/230 ratios provided by NanoDrop Technologies. For the quantitative real-time reverse transcription polymerase chain reaction (RT-PCR) analysis, the first strand cDNA was synthesized from $500 \mathrm{ng}$ of total RNA using a PrimeScript ${ }^{\text {tm }}$ Kit (TaKaRa, Bio Inc, 
China) following the manufacturer's protocols. Real-time RT-PCR was performed using the SYBR ${ }^{\circ}$ Premix Ex Taq II (TaKaRa, Bio Inc, China). Real-time RT-PCR analysis was conducted on a $20 \mu \mathrm{l}$ mixture containing $10.0 \mu \mathrm{l}$ SYBR $^{\circ}$ Premix Ex Taq ${ }^{\text {Tit }}$ II, $2.0 \mu \mathrm{l}$ diluted cDNA and $0.8 \mu \mathrm{l}$ of the forward and reverse primers. The amplification was completed with the cycling parameters of $95^{\circ} \mathrm{C}$ for $1 \mathrm{~min}$, followed by 45 cycles at $95^{\circ} \mathrm{C}$ for $10 \mathrm{~s}, 48^{\circ} \mathrm{C}$ for $30 \mathrm{~s}$ and $72^{\circ} \mathrm{C}$ for $20 \mathrm{~s}$. The internal control (reference gene) used was the Ubi3 (AY486137.1) gene, as done previously [19]. The primer sequences used for real-time RT-PCR are shown in Additional file 12 Table S2. The relative expression levels of each gene were calculated using the Delta-Delta Ct method [20]. All samples were obtained in triplicate and each treatment had at least three independent biological replicates.

\section{Analysis of major carotenoid contents in WT, TRV/00 and gene silenced pepper fruits}

Using the method of Lopez-Raez et al. [21], the carotenoids were extracted and identified. Specifically, a $5.0 \mathrm{~g}$ sample of pericarp tissue was extracted with $5 \mathrm{ml}$ of acetone containing $0.1 \%$ butylated hydrox-ytoluene (BHT). After shaking and incubation on ice in the dark for $10 \mathrm{~min}$, the mixture was centrifuged at $3500 \mathrm{rpm}$ for $10 \mathrm{~min}$ at room temperature and the extract was transferred to a clean tube. Samples were re-extracted twice with $5.0 \mathrm{ml}$ of acetone containing $0.1 \%$ BHT. Pooled extracts were dried under a nitrogen flow, and the tubes were sealed and stored at $-20^{\circ} \mathrm{C}$ until high pressure liquid chromatography (HPLC) analysis. HPLC was performed as described previously [22]. For HPLC, samples $(20 \mu \mathrm{L})$ were analyzed on a shim-pack VP-ODS C-18 HPLC column $(5 \mu \mathrm{m}, 150 \mathrm{~mm} \times 4.6 \mathrm{~mm})$. The eluent consisted of acetonitrile:2-propanol:water in a ratio of 39:53:8 (A) and acetonitrile:2-propanol in a ratio of 60:40 (B). The gradient profile was $0-30 \mathrm{~min}$ from 0 to $100 \% \mathrm{~B}$. The flow rate was set at $0.3 \mathrm{~mL} / \mathrm{min}$ and the column temperature at $40^{\circ} \mathrm{C}$. Standard solutions of $\beta$-carotene, $\beta$-cryptoxanthin, zeaxanthin and capsanthin $(0.001-0.1 \mathrm{mg} / \mathrm{mL})$ were used to make calibration curves at $454 \mathrm{~nm}$. The carotenoids were identified by their absorption spectra as captured by the photodiode array detector, and HPLC retention times in comparison with authentic standards. In addition, samples were spiked with standards to verify the identity of sample peaks with similar retention times. $\beta$-carotene was obtained from Toshima Kita-ku(Tokyo, Japan); zeaxanthin was obtained from Shanghai yuanye biological technology Co. (China); $\beta$-cryptoxanthin and capsanthin were purchased from Extrasynthèse (Genay, France), and they were used as authentic standards. All standards were handled under low light conditions on ice. Standard solutions of $\beta$ carotene, $\beta$-cryptoxanthin, zeaxanthin and capsanthin standard were in methanol:acetonitrile $(1: 1, \mathrm{~V} / \mathrm{V})$. Aliquots were diluted in methanol:acetonitrile (1:1) to provide standard concentrations [22,23].

\section{Additional files}

Additional file 1: Table S1. The measurement of color variation in yellow, deep yellow and orange fruits between the phenotypes. WT: fruits not injected; TRV/00: fruits injected tempt vector; the vectors that tobacco rattle virus (TRV) carried target gene. They are demarking TRV/ Psy/Lcyb/Crtz/CCS, TRV/Psy/Lcyb/Crtz, TRV/CCs, TRV/Psy/Lcyb, TRV/Crtz, TRV/ LCyb, and TRV/Psy. The mean values were determined by Chroma meter (Made in Japan). The chroma of WT fruits were used as the reference when determining $\Delta \mathrm{L}, \Delta \mathrm{a}$ and $\Delta \mathrm{b}$. Every research matierial is three independent biological replicates

Additional file 2: Figure S1. Resolution of Capsicum pericarp carotenoids by UPLC. Carotenoids were detected by absorption at $454 \mathrm{~nm}$ following separation on a C18 column as described in the methods. (A) Standards (each at 10 ppm): capsanthin (7.36 min), zeaxanthin (7.93 min), $\beta$-cryptoxanthin (10.88 min) and $\beta$-carotene (23.04 min). (B) the extracted pericarp in WT.

Additional file 3: Figure S2. Resolution of Capsicum pericarp carotenoids by UPLC. Carotenoids were detected by absorption at $454 \mathrm{~nm}$ following separation on a C18 column as described in the methods. (A) Standards (each at $10 \mathrm{ppm}$ ): capsanthin (7.36 min), zeaxanthin (7.93 min), $\beta$-cryptoxanthin (10.88 min) and $\beta$-carotene (23.04 min). (B) the extracted pericarp in TRV/00.

Additional file 4: Figure S3. Resolution of Capsicum pericarp carotenoids by UPLC. Carotenoids were detected by absorption at $454 \mathrm{~nm}$ following separation on a C18 column as described in the methods. (A) Standards (each at 10 ppm): capsanthin (7.36 min), zeaxanthin (7.93 min), $\beta$-cryptoxanthin (10.88 min) and $\beta$-carotene (23.04 min). (B) the extracted pericarp in TRV/CCS.

Additional file 5: Figure S4. Resolution of Capsicum pericarp carotenoids by UPLC. Carotenoids were detected by absorption at $454 \mathrm{~nm}$ following separation on a C18 column as described in the methods. (A) Standards (each at $10 \mathrm{ppm})$ : capsanthin (7.36 min), zeaxanthin (7.93 min), $\beta$-cryptoxanthin (10.88 min) and $\beta$-carotene (23.04 min). (B) the extracted pericarp in TRV/Psy.

Additional file 6: Figure S5. Resolution of Capsicum pericarp carotenoids by UPLC. Carotenoids were detected by absorption at $454 \mathrm{~nm}$ following separation on a C18 column as described in the methods. (A) Standards (each at 10 ppm): capsanthin (7.36 min), zeaxanthin (7.93 min), $\beta$-cryptoxanthin (10.88 min) and $\beta$-carotene (23.04 min). (B) the extracted pericarp in TRV/Lcyb.

Additional file 7: Figure S6. Resolution of Capsicum pericarp carotenoids by UPLC. Carotenoids were detected by absorption at $454 \mathrm{~nm}$ following separation on a C18 column as described in the methods. (A) Standards (each at $10 \mathrm{ppm}$ ): capsanthin (7.36 min), zeaxanthin (7.93 min), $\beta$-cryptoxanthin (10.88 min) and $\beta$-carotene (23.04 min). (B) the extracted pericarp in TRV/Crtz.

Additional file 8: Figure S7. Resolution of Capsicum pericarp carotenoids by UPLC. Carotenoids were detected by absorption at $454 \mathrm{~nm}$ following separation on a C18 column as described in the methods. (A) Standards (each at $10 \mathrm{ppm})$ : capsanthin (7.36 min), zeaxanthin (7.93 min), $\beta$-cryptoxanthin (10.88 $\mathrm{min}$ ) and $\beta$-carotene (23.04 min). (B) the extracted pericarp in TRV/Psy/Lcyb.

Additional file 9: Figure S8. Resolution of Capsicum pericarp carotenoids by UPLC. Carotenoids were detected by absorption at $454 \mathrm{~nm}$ following separation on a C18 column as described in the methods. (A) Standards (each at 10 ppm): capsanthin (7.36 min), zeaxanthin (7.93 min), $\beta$-cryptoxanthin (10.88 min) and $\beta$-carotene (23.04 min). (B) the extracted pericarp in TRV/Psy/Lcyb/Crtz.

Additional file 10: Figure S9. Resolution of Capsicum pericarp carotenoids by UPLC. Carotenoids were detected by absorption at $454 \mathrm{~nm}$ following separation on a C18 column as described in the methods. (A) Standards (each at 10 ppm): capsanthin (7.36 min), zeaxanthin (7.93 min), 
$\beta$-cryptoxanthin (10.88 $\mathrm{min}$ ) and $\beta$-carotene (23.04 min). (B) the extracted pericarp in TRV/Psy/Lcyb/Crtz/Ccs.

Additional file 11: Figure S10. Schematic representation of TRV. LB: left borders of the T-DNA; RB: right borders of the T-DNA; $2 \times 35 \mathrm{~S}$ : two copies of the cauliflower mosaic virus 355 promoter; CP: coat protein; RdRp: RNA-dependent RNA polymerase; MP: movement protein; $16 \mathrm{~K}: 16$ KDa protein; R: ribozyme; N: nos-terminator; and MCS: multiple cloning sites.

Additional file 12: Table S2. Primers were used in quantitative real-time RT-PCR and plasmid construction. Psy: Phytoene synthase gene; Crtz: $\beta$-carotene hydroxylase gene; Lcyb: Lycopene- $\beta$-cyclase gene; Ccs: Capsanthin/capsorubin synthase gene; Ubi3 was used as internal control (reference gene); underlined GGATCC is BamH I endonuclease site, underlined GGTACC is Kpn I endonuclease site.

Additional file 13: Figure S11. Schematic representation of recombinant TRV vectors carrying target genes. From the BamHI and Kpnl restrictive endonuclease sites, and joining the target gene fragments and TRV vector together, they were pTRV2/CcS, pTRV2/Psy, pTRV2/Lcyb and pTRV2/Crtz.

Additional file 14: Figure S12. Detection of cloned PMD19-T vectors by PCR. The target gene fragments were detected by colony PCR and ligated into the cloning vector pMD19-T before being transformed into $E$. coll DH5a. As determined from the sequencing, cloned vectors were developed successfully that contained fragments of the Ccs, Psy, Lcyb and Crtz genes.

Additional file 15: Figure S13. Construction of TRV expression vectors. The target gene fragments were detected by colony PCR and gene sequences that showed the TRV expression vectors carrying fragments of the Ccs, Psy, Lcyb and Crtz genes were successfully constructed.

\section{Abbreviations}

TRV: Tobacco rattle virus; VIGS: Virus-induced gene silencing

PTGS: Posttranscriptional gene silencing; Ccs: Capsanthin-capsorubin synthase; Crtz: $\beta$-carotene hydroxylase; Lcyb: Lycopene- $\beta$-cyclase; Psy: Phytoene synthase; Ubiz: Ubiquitin conjugating protein; PTGS: Posttranscriptional gene silencing; DAA: Day after anthesis; HPLC: High pressure liquid chromatography; BHT: Butylated hydrox-ytoluene; GGPP: Geranylgeranyl diphospahate.

\section{Competing interests}

The authors declare that they have no competing interests.

\section{Authors' contributions}

ZHG, SLT and LL designed the research, analyzed data and drafted the manuscript. SLT and WGC designed primers for the RT-qPCR assays and collected virus samples. SLT and ZHG carried out virus vector construction and VIGS technology. LL and WGC carried out the optimization of the RT-qPCR assays, contributed to the design of the study, primer design, sample collection, statistical analysis and design of the duplex RT-qPCR protocol. LL and SNMS extracted RNA from pepper fruits. SLT wrote the paper, and all the authors read and approved the final manuscript.

\section{Acknowledgements}

The following organizations are acknowledged for their financial support: National Natural Science Foundation of China (No. 31272163), Zhejiang Province Key Scientific and Technological Project (2011C02001), "The Twelfth Five-Year" Plan of National Science and Technology in Rural Areas (No.2011BAD12B03) and the Shaanxi Provincial Science and Technology Coordinating Innovative Engineering Project (2012KTCL02-09). Language editing was done by MogoEdit (Xi'an) Company Ltd. (http://www.mogoedit.com)

\section{Author details}

${ }^{1}$ College of Horticulture, Northwest A\&F University, Yangling, Shaanxi 712100, P. R. China. ${ }^{2}$ Huanghuai University, Zhumadian, Henan 463000, P. R. China ${ }^{3}$ Institute of Vegetables, Hangzhou Academy of Agricultural Sciences, Hangzhou, Zhejiang 310024, P. R. China. ${ }^{4}$ Department of Horticulture, Faculty of Agriculture, Gomal University Dera Ismail Khan, Dera Ismail Khan, Pakistan.

\section{References}

1. MacFarlane SA: Molecular biology of the tobraviruses. J Gen Virol 1999, 80:2799-2807

2. Becker A, Lange M: VIGS-genomics goes functional. Trends Plant Sci 2010 15:1-4.

3. Baulcombe DC: Fast forward genetics based on virus-induced gene silencing Curr Opin Plant Biol 1999, 2:109-113.

4. Paroo Z, Liu Q, Wang X: Biochemical mechanisms of the RNA-induced silencing complex. Cell Res 2007, 17:187-194.

5. Ha SH, Kim JB, Park JS, Lee SW, Cho KJ: A comparison of the carotenoid accumulation in Capsicum varieties that show different ripening colours: deletion of the capsanthin-capsorubin synthase gene is not a prerequisite for the formation of a yellow pepper. J Exp Bot 2007, 58(12):3135-3144.

6. Guzman I, Hamby S, Romero J, W. Bosland P, A. O'Connell M: Variability of carotenoid biosynthesis in orange colored Capsicum spp. Plant Sci 2010 179:49-59.

7. DellaPenna D, Pogson BJ: Vitamin synthesis in plants: Tocopherols and carotenoids. Annu Rev Plant Biol 2006, 57:711-738.

8. Ronen G, Cohen M, Zamir D, Hirschberg J: Regulation of carotenoid biosynthesis during tomato fruit development: Expression of the gene for lycopene epsilon-cyclase is down-regulated during ripening and is elevated in the mutant Delta. Plant J 1999, 17:341-351.

9. Moehs CP, Tian L, Osteryoung KW, DellaPenna D: Analysis of carotenoid biosynthetic gene expression during marigold petal development. Plant $\mathrm{Mol}$ Biol 2001, 45:281-293.

10. Lefebvre V, Kuntz M, Camara B, Palloix A: The capsanthin-capsorubin synthase gene: a candidate gene for the $y$ locus controlling the red fruit colour in pepper. Plant Mol Biol 1998, 36:785-789.

11. Thorup TA, Tanyolac B, Livingstone KD, Popovsky S, Paran I, Jahn M: Candidate gene analysis of organ pigmentation loci in the Solanaceae. Proc Nati Acad Sci U S A 2000, 97:11192-11197.

12. Fu DQ, Zhu BZ, Zhu HL, Jiang $W B$, Luo YB: Virus-induced gene silencing in tomato fruit. Plant J 2005, 43:299-308.

13. Wege S, Scholz A, Gleissberg S, Becker A: Highly efficient virus-induced gene silencing (VIGS) in California poppy (Eschscholzia californica): an evaluation of VIGS as a strategy to obtain functional data from non-model plants. Ann Bot 2007, 100:641-649.

14. Yamagishi N, Yoshikawa N: Virus-induced gene silencing in soybean seeds and the emergence stage of soybean plants with Apple latent spherical virus vectors. Plant Mol Biol 2009, 75:15-24.

15. Holzberg S, Brosio P, Gross C, Pogue GP: Barley stripe mosaic virus-induced gene silencing in a monocot plant. Plant J 2002, 30:315-327.

16. Kormos J, Kormos K: Die genetischen typen der carotenoid-systeme der paprikafrucht. Act Bot Acad Sci Hun 1960, 6:305-319.

17. Wang JE, Liu KK, Li DW, Zhang YL, Zhao Q, He YM, Gong ZH: A novel peroxidase $\mathrm{CaPOD}$ gene of pepper is involved in defense responses to phytophtoracapsici Infection as well as abiotic stress tolerance. Int J Mol Sci 2013, 14:3158-3177.

18. Wang HM, Yin WC, Wang CK, To KY: Isolation of functional RNA from different tissues of tomato suitable for developmental profiling by microarray analysis. Bot Stud 2009, 50:115-125

19. Wan HJ, Yuan W, Ruan MY, Ye QJ, Wang RQ, Li ZM, Zhou GZ, Yao ZP, Zhao J Liu SJ, Yang YJ: Identification of reference genes for reverse transcription quantitative real-time PCR normalization in pepper (Capsicum annuum L.). Biochem Bioph Res Co 2011, 416:24-30.

20. Livak KJ, Schmittgen TD: Analysis of relative gene expression data using real-time quantitative PCR and the 2(T)(-Delta Delta C) method. Methods 2001, 25:402-408.

21. Lopez-RaezJA CT, Mulder P, Kohlen W, Bino R, Levinl BH: Susceptibility of the tomato mutant high pigment-2dg(hp-2dg) to Orobanche spp. infection. J Agric Food Chem 2008, 56:6326-6332

22. Rodriguez-Uribe L, Guzman I, Rajapakse W, Richins RD, O'Connell MA: Carotenoid accumulation in orange-pigmented Capsicum annuum fruit, regulated at multiple levels. J Exp Bot 2012, 63(1):517-526.

23. Wall MM, Waddell CA, Bosland PW: Variation in beta-carotene and total carotenoid content in fruits of Capsicum. Hortscience 2001, 36(4):746-749.

doi:10.1186/s12870-014-0314-3

Cite this article as: Tian et al:: Effects of silencing key genes in the capsanthin biosynthetic pathway on fruit color of detached pepper fruits. BMC Plant Biology 2014 14:314. 